\title{
Gestión ambiental de una empresa minera de yeso en Manaure, Colombia
}

\author{
Katerin P. Salcedo, Carlos M. Tapia y Danny D. López \\ Facultad de Ingeniería, Universidad de La Guajira, Km. 5 salida a Maicao, Riohacha-Colombia. \\ (correo-e: ksalcedo@uniguajira.edu.co; cmtapias@uniguajira.edu.co; dlopezj@uniguajira.edu.co).
}

Recibido Mar. 9, 2021; Aceptado May. 12, 2021; Versión final Jun. 22, 2021, Publicado Oct. 2021

\begin{abstract}
Resumen
El propósito de esta investigación es analizar la gestión ambiental en la empresa minera "El Porvenir" en Manaure, Colombia. Se trata de un estudio descriptivo, no experimental y transversal. La población muestreada la componen 31 trabajadores del área de producción de la mina "El Porvenir". Se emplea la técnica de observación aplicando instrumentos como la matriz DOFA y una lista de chequeo. Como resultado, el diagnóstico ambiental evidencia graves problemas ambientales producto del desconocimiento de la legislación ambiental aplicable a los procesos mineros que se llevan a cabo en la empresa minera "EI Porvenir". Se concluye que la matriz DOFA y la lista de chequeo son instrumentos eficaces para determinar los impactos generados a los componentes ambientales y que la gestión ambiental en la empresa minera "EI Porvenir" puede contribuir al mejoramiento de los procesos y/o actividades mineras que se llevan a cabo en esta empresa.
\end{abstract}

Palabras clave: gestión ambiental; yeso; diagnostico; empresa minera; lista de chequeo

\section{Environmental management of a gypsum mining company in Manaure, Colombia}

\begin{abstract}
The purpose of this research study is to assess the environmental management practices of the mining company El Porvenir in Manaure, Colombia. This is a descriptive, non-experimental, and cross-sectional study. The sampled population is composed of 31 workers from the production area of El Porvenir mine. The observation technique is used and the instruments applied include a SWOT matrix and a checklist. As a result, the environmental diagnosis shows serious environmental issues due to lack of knowledge of the environmental legislation that applies to the mining processes performed by the mining company El Porvenir. It is concluded that the SWOT matrix and a checklist are effective instruments to assess environmental impact and that El Porvenir's environmental management can contribute to improving the mining processes and/or activities performed by this company.
\end{abstract}

Keywords: environmental management; gypsum; diagnosis; mining company; checklist 


\section{INTRODUCCIÓN}

Durante muchos años se mantuvo el concepto en el cual la protección ambiental resultaba excesivamente costosa y frenaba el desarrollo (Cicatiello et al., 2020). Hoy día se demuestra que la problemática y las necesidades tanto presentes como futuras obligan a efectuar un desarrollo sostenible y equilibrado, protegiendo al ambiente y haciendo uso racional de los recursos naturales tanto renovables como no renovables (Gunnarsdottir, et al., 2021). La creciente sensibilidad que ha desarrollado la humanidad respecto a la importancia de la protección del medio ambiente ha aumentado el interés por implementar estrategias que permitan comprender y reaccionar frente a los efectos que se generan a partir del desarrollo económico fundamentado en la utilización y aprovechamiento de los recursos naturales (Figge y Hahn, 2020). Estos efectos podrían ocasionar graves consecuencias medioambientales, las cuales se originan a partir del momento en el que se inicia con el proceso de extracción de minerales y materias primas; por lo que no se precisan los impactos que producen en el entorno, para ello tendrían que evaluarse factores como el uso de los recursos naturales en las regiones extractivas.

Las regiones extractivas son asentamientos vulnerables que dependen excesivamente de la explotación de los recursos naturales. El destino de estas regiones está estrechamente asociado con el de las principales industrias extractivas debido a las estrechas bases económicas, el aislamiento geográfico y la ausencia de alternativas realistas para el desarrollo diversificado (Lo, 2020). Las regiones extractivas que no transforman rápidamente sus economías a menudo sufren declive y pérdida de población cuando los recursos de los que dependen se agotan o la explotación ya no se considera económica (Ross, 2019). En consecuencia, la transformación hacia una economía más diversificada y sostenible ha sido un tema central en la gobernanza extractiva a nivel mundial (Li et al., 2015).

En este sentido, se deben considerar estrategias que potencien el desarrollo económico de las empresas extractivistas o mineras, pero cuyas bases estén fundamentadas en un desarrollo sostenible y equilibrado entre lo - económico, ambiental y social -, por medio de herramientas como la gestión ambiental. La adopción de Sistemas de Gestión Ambiental (SGA) puede ser una herramienta eficaz para que las organizaciones aborden los problemas económicos, sociales y ambientales y parece ser un medio viable para desarrollar objetivos comerciales y mejorar las actividades de responsabilidad ambiental empresarial (Ikram et al., 2019). De este modo, los SGA en las empresas mineras permiten orientar sus decisiones y esfuerzos para alcanzar y demostrar un buen desempeño en el ámbito ambiental, creando compromisos de cumplimiento según lo dispuesto por legislación ambiental aplicable y controlando el impacto producto sus actividades. En este contexto, surge el otorgamiento de licencias ambientales, con el reto de conciliar la realización de todas las actividades de una manera sostenible (Fernandes et al., 2017).

En efecto, el análisis de un SGA implica evaluar los objetivos ambientales corporativos y las políticas ambientales, asignar responsabilidades finales y determinar las acciones de implementación del SGA. Sin embargo, muchas empresas comienzan por error la implementación inmediatamente después de un diagnóstico ambiental inicial sin revisar críticamente los objetivos y las políticas. Por lo tanto, la alta dirección debe primero refinar los objetivos y acciones ambientales pertinentes, y debe realizar múltiples revisiones ambientales antes de implementar el SGA. Además, es práctico para la administración desarrollar algún tipo de esquema de informes ambientales en esta etapa para mantener informadas a las partes interesadas sobre la estructura general del SGA, sus objetivos y las responsabilidades del personal seleccionado. A partir de este punto, la responsabilidad recae en los mineros y operadores de minas para garantizar que las acciones responsables se lleven a cabo dentro de las limitaciones de los compromisos de la empresa (Hilson y Nayee, 2002). Las prácticas de gestión ambiental se pueden dividir en dos categorías principales según el carácter obligatorio o voluntario de las fuerzas impulsoras. La primera categoría engloba las prácticas de gestión ambiental de las empresas mineras resultantes de la presión de los instrumentos de "mando y control" y "basados en el mercado". La segunda categoría incluye aquellas prácticas de gestión ambiental que la industria minera adopta de manera voluntaria (Nikolaou y Evangelinos, 2010).

Además, otra distinción útil para las prácticas de gestión ambiental es el período en el que se aplican estas prácticas, es decir, las prácticas de gestión ambiental ex ante o ex post. Las prácticas de gestión ambiental de las empresas mineras para restaurar el entorno físico se aplican al final del proceso productivo y podrían considerarse ex post. Esta categoría incluye prácticas de gestión ambiental basadas principalmente en la idea de rehabilitación del daño ambiental minero y es principalmente resultado del régimen regulatorio, por lo que pueden ser consideradas acciones reactivas. La otra categoría abarca las prácticas de gestión ambiental que adoptan las industrias mineras y mineras para mantener un buen nivel de calidad ambiental y puede clasificarse como una práctica ex ante y puede considerarse más proactiva (Nikolaou y Evangelinos, 2010).

El yeso es una roca blanda con baja resistencia y alta propensión a la deformación plástica. Sus propiedades mecánicas son relevantes en una amplia gama de aplicaciones técnicas (por ejemplo, excavación de túneles, 
evaluación de la estabilidad de canteras subterráneas, acumulación de petróleo y gas). Los micromecanismos involucrados en la deformación de la roca de yeso han interesado durante mucho tiempo al mundo científico y aún no se comprenden completamente y requiere una atención específica a la evaluación de la gestión ambiental en este tipo de minería (Caselle et al., 2020). De este modo, la presente investigación estudia la gestión ambiental en empresas mineras que producen yeso. Actualmente, la disponibilidad de yeso en el mercado es relativamente amplia, las principales fuentes son el yeso natural extraído en ciertas regiones y varios tipos de yeso de desecho que aparecen como subproductos de algunas actividades industriales, por ejemplo, desulfuración de gases de combustión, fosfo-titano y boro-yeso. Sin embargo, a pesar de la larga historia de su uso, el yeso tiene actualmente solo una aplicación limitada en la industria de la construcción, principalmente en forma de placas de yeso o yesos interiores (Fořt y Černý, 2018).

Los análisis del impacto ambiental de la producción de yeso publicados hasta la fecha son relativamente escasos y en su mayoría sufrieron la falta de datos primarios. Jimenéz-Rivero y García-Navarro (2016) en uno de los pocos estudios sobre el ciclo de vida del yeso utilizaron datos genéricos derivados de los estudios de caso de materiales de construcción similares. Sin embargo, los problemas relacionados con la ausencia de datos primarios también se notaron para los materiales de construcción analizados con mayor frecuencia, como el hormigón, debido a la falta de datos primarios sobre el hormigón en parte a la falta de voluntad de los sujetos industriales para proporcionar datos reales sobre los impactos ambientales de sus actividades (Dong et al., 2015). Por lo tanto, con el fin de proporcionar una visión global de los impactos ambientales en la minería de yeso, se deben tener en cuenta los factores locales, como la composición de la combinación energética, la base de combustible, las tecnologías de fabricación y la legislación ambiental vigente.

Partiendo de la importancia que tienen los SGA dentro de la industria extractiva, en este trabajo se presenta el análisis de la gestión ambiental en la empresa minera "El Porvenir" dedicada a la explotación artesanal de yeso en Manaure (Colombia), como estrategia para lograr una mejora en los procedimientos, responsabilidades y procesos necesarios para prevenir los impactos ambientales, económicos y sociales de este tipo de minería. Así mismo, esta investigación es relevante porque ayuda a implementar un nuevo estándar de minería en antiguas y nuevas empresas que se dediquen a la explotación de yeso a nivel local, nacional e internacional.

\section{METODOLOGIA}

La presente investigación es de carácter evaluativa, ya que permite a los investigadores realizar una exploración en busca de patrones significativos sobre la problemática estudiada, a partir de estos resultados los autores sustentan las explicaciones acerca de los factores influyentes en la problemática, identificar las causas y consecuencias de los sucesos y la relación entre estos con las variables estudiadas (Balnaves y Caputi, 2001), de esta manera en el presente estudio se determina cuáles son los factores ambientales que se ven a afectados con la extracción y el tratamiento del yeso por parte de la empresa "El Porvenir"; así como, recolectar la información necesaria para realizar un análisis sobre qué tan favorable seria implementar un SGA para darle solución a las problemáticas ambientales presentes en la mina; con un diseño de investigación no experimental, porque no existe manipulación deliberada de la variable estudiada, solo se observa y analiza en su entorno; de corte transeccional, ya que la investigación se desarrolla en un periodo de tiempo previamente definido (Hernández et al., 2010). La población estudiada estuvo conformada por 31 trabajadores de la empresa minera "El Porvenir" en Manaure, (Colombia), la zona de estudio comprendió la empresa en mención y se extendió hasta su área de influencia. En la Tabla 1 se observa la población, que fue de carácter finita, correspondiente a los trabajadores por área productiva en la que laboran.

Tabla 1: Número de áreas productivas en la empresa minera.

\begin{tabular}{|l|c|}
\hline Áreas productivas & Número de trabajadores \\
\hline Extracción del yeso & 7 \\
\hline Transporte del yeso & 3 \\
\hline Almacenamiento del yeso & 7 \\
\hline Lavado del yeso & 6 \\
\hline Triturado del yeso & 8 \\
\hline Total & 31 \\
\hline
\end{tabular}

Respecto a las fuentes de información, se utilizaron fuentes primarias, y secundarias, donde las fuentes primarias corresponden a visitas técnicas en campo, donde se aplicó los instrumentos: matriz DOFA (Nikolaou y Evangelinos, 2010), lista de chequeo (Molléri et al., 2020; Creswell, 2003), aplicados para la recolección de información, y las fuentes secundarias son las fuentes bibliográficas (libros y artículos científicos). De acuerdo con la lista de chequeo, esta estuvo compuesta por los factores ambientales receptores de impactos y sus afectaciones, causadas por la explotación de yeso. Por su parte, la matriz DOFA se utilizó para realizar un 
diagnóstico ambiental en la mina "El porvenir". Cabe resaltar que no se utilizó procedimientos estadísticos formales, en su lugar se definió el cumplimiento o no de la normativa ambiental colombiana.

Para el desarrollo del diagnóstico ambiental de la empresa minera "El Porvenir" se realizaron visitas técnicas a las zonas de operación, donde se efectuaron análisis de carácter descriptivo sobre los factores que impactan negativamente los recursos naturales presentes en el área de influencia de la empresa, estas visitas permitieron la aplicación de los instrumentos utilizados en la recolección de información, la información obtenida por estos instrumentos se analizó a detalle permitiendo identificar el estado ambiental de la empresa. Posteriormente, se establecieron una serie de estrategias ambientales encaminadas a la prevención, mitigación y compensación de los daños ambientales identificados en la zona estudiada; además, se definieron que instrumentos ambientales debería aplicar la empresa para empoderar a sus trabajadores en el cuidado ambiental.

En cuanto, a la Validez y confiabilidad de los instrumentos utilizados para la investigación se evaluó la pertinencia en relación con su alcance, contenido, efectividad, coherencia, redacción, entre otros y se validó por medio del criterio de consulta técnica a expertos en las áreas de metodología de la investigación, en ciencias ambientales y minería de yeso y la confiabilidad no fue medida, por cuanto este estudio está sujeto a criterios propios como investigadores tanto en la observación en campo como para la selección de material documental.

\section{RESULTADOS Y DISCUSIÓN}

El procesamiento del material inicia en la cantera mediante la extracción de la materia prima y finaliza después de la producción del yeso (Fig. 1). Los métodos de extracción de yeso que implementa la empresa "El Porvenir" se basan en la utilización de procedimientos y herramientas convencionales, este proceso se divide en dos bloques; en el primer bloque se realiza la identificación del área a intervenir, luego se procede con el descapotamiento y perforación del suelo, luego, el material extraído se carga en camiones y se lleva a la zona de almacenamiento donde se apilan hasta tener una cantidad considerablemente grande que justifique económicamente el lavado del material, en el segundo bloque se realiza el lavado del yeso en las piscinas de agua, este lavado se aplica con el objetivo de eliminar las impurezas que pueda tener el mineral, una vez finalizado el proceso de lavado, se procede a esparcir el yeso en un área delimitada, y con una buena incidencia de los rayos solares para que este se seque, para finalizar se recoge el material seco y se tritura y empaca para posteriormente ser distribuido; la producción anual de yeso en esta cantera oscila entre 30 y 45 toneladas.

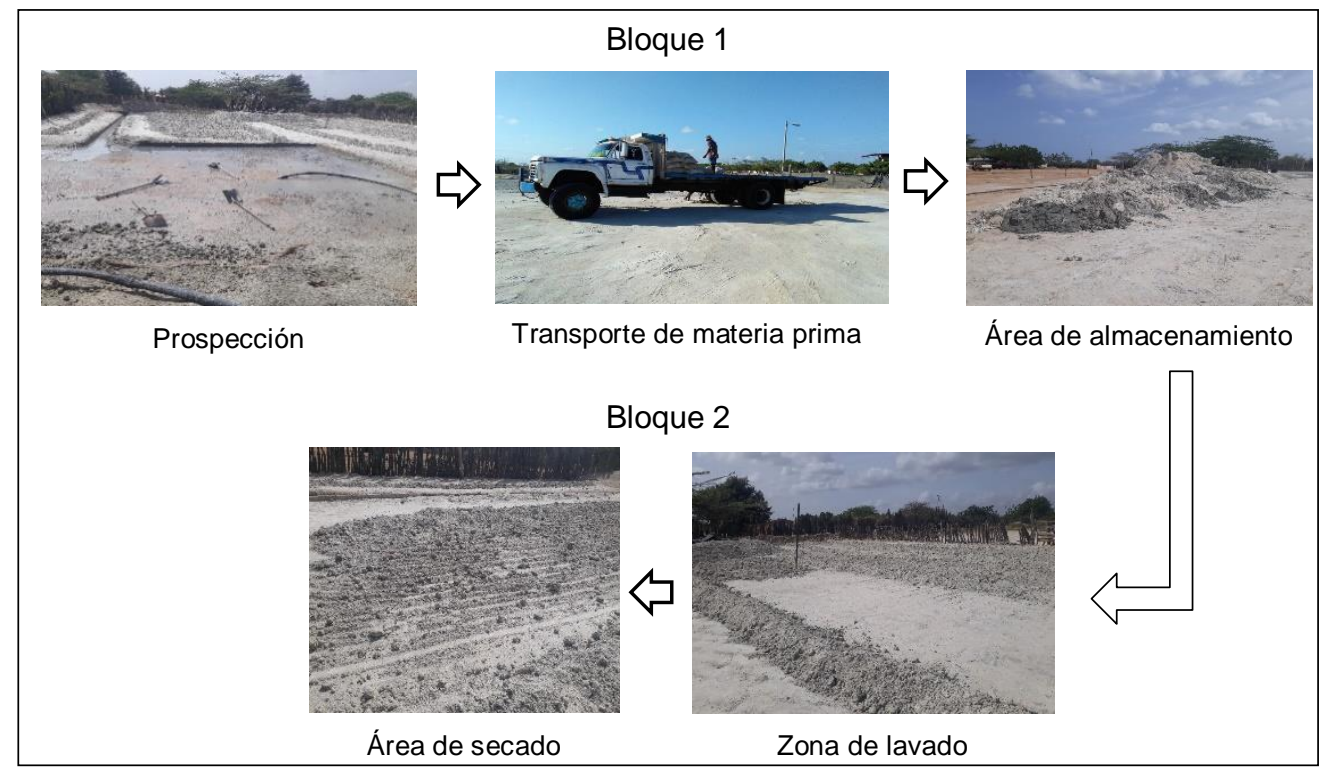

Fig. 1: Procesamiento de yeso en la empresa minera "El Porvenir"

Cabe resaltar que este procesamiento corresponde al mismo que reporta López (2010), por lo que es indispensable que este tipo de canteras implemente maquinaria y/o atraiga la inversión privada para aumentar la producción, darles capacitación a los mineros tradicionales para el manejo adecuado de las máquinas e involucrarlos en proyectos que potencien su actividad extractiva. Es imperioso emplear nuevos métodos industriales para una explotación y procesamiento del yeso más tecnificado y con altos estándares de calidad y seguridad. 
A partir del procesamiento del yeso, se realizó un análisis de los aspectos e impactos ambientales generados a partir de la forma en que se ejecutan las actividades en la empresa "El Porvenir", este análisis indica que la empresa no utiliza una maquinaria ecológica con bajo consumo hídrico y bajas emisiones de dióxido de carbono y otros gases clasificados como gases de efecto invernadero; las fuentes contamines móviles dentro de la empresa (vehículos) además de emitir contaminantes atmosféricos, generan contaminación auditiva durante el cargue, trasporte y descargue de los materiales propios de esta minería, de acuerdo con (CacildaAndre et al., 2019) este ruido puede ocasionar la migración de la fauna presente en la zona de influencia de la mina, propiciando un desequilibrio ecológico. Se observó también que la empresa cuenta con un pozo el cual se concibió y funciona de forma desarticulada con lo estipulado en el Decreto Único Reglamentario del Sector Ambiente y Desarrollo Sostenible 1076 de 2015 en Colombia, en el que se establece que el uso y aprovechamiento del agua deberá darse a partir de restricciones y limitaciones que estén en favor del interés general de la comunidad y de ese modo asegurar el aprovechamiento de las aguas para todos los usuarios.

Con respecto a los componentes abióticos, se observaron alteraciones en la calidad y las características de los suelos presentes en el área de trabajo de la empresa, estas alteraciones pueden haberse producido por derrame de sustancias químicas tales como: lubricantes, ácidos y combustibles, y/o por la forma artesanal en que la empresa explota el suelo para extraer el yeso. También se observó un vertimiento puntual al suelo de las aguas sin tratar utilizadas para el lavado del mineral, esta última es una actividad critica teniendo en cuenta que en el área de la mina circula agua subterránea; y podría generarse una contaminación por las impurezas presentes en el yeso (Ricci et al., 2000), aportándole características toxicológicas a estas aguas subterráneas (He et al., 2021).

Por otro lado, se validó que la empresa "El Porvenir" tiene conocimiento acerca de la existencia de normas para la protección y conservación del medio ambiente y de minería, pero por medio de una revisión se encontró que está no tiene los permisos necesarios para desarrollar sus actividades y que no cuenta con planes para la adecuación de los ecosistemas ya intervenidos; inclusive no existe un plan de acción interno que le permita a la empresa disponer de forma adecuada y aprovechar los residuos sólidos que genera diariamente en cada uno de los procesos que lleva a cabo. Si bien es cierto que los impactos que se ocasionan en la explotación del yeso son menores a los generados en la producción de cemento (Guo y Shi, 2008), por ello, estos deben ser atendidos con prioridad debido a que si se prolongan en el tiempo pueden llegar hacer irreversibles.

La identificación de la síntesis ambiental se desarrolló por medio de la aplicación de herramientas que permitieron obtener un diagnóstico ambiental mucho más amplio y preciso; con la matriz DOFA (Tabla 2). Lozano y Valles (2007) afirman que "el análisis DOFA es ampliamente reconocido y constituye una base importante para conocer la situación y diseñar procedimientos futuros que se vean necesarios para pensar de manera estratégica". Sin embargo, el uso de este método da lugar a importantes ventajas y desventajas. Las ventajas, por ejemplo, pueden incluir la idea de que este método es muy simple y todos pueden usarlo sin tener conocimientos avanzados o soporte técnico externo. Las desventajas se refieren a su carácter simplista, estático y subjetivo. Sin embargo, este estudio extrae información de campo en la empresa "EI Porvenir", por lo que los datos son reales y superó la subjetividad de las respuestas de los trabajadores que laboran en la empresa.

De este modo, al aplicar la matriz DOFA se encontró entre otras cosas, que existe un desinterés por parte de la empresa para diseñar e implementar iniciativas que le permitan solucionar las problemáticas ambientales que está experimenta, y que existe el riesgo de comprometer de manera critica la calidad de los ecosistemas que están siendo intervenidos en la explotación, cabe resaltar que estos riesgos son el resultado de malas decisiones e inadecuadas prácticas ambientales que se han puesto en práctica en la mina "El Porvenir". Así mismo, y para unificar los criterios obtenidos con la matriz DOFA, se aplicó una lista de chequeo, que son utilizadas tanto para realizar como para evaluar diferentes tipos de estudios empíricos, como experimentos y estudios de caso (Stavru, 2014; Canter y Kamath, 1995), en el caso del presente estudio, se aplicó una lista de chequeo donde se relaciona los componentes o factores ambientales receptores de impacto en el desarrollo de esta minería y las actividades susceptibles de producir impacto (Tabla 3), donde se observa que los componentes flora y fauna, territorio y alteración al paisaje están siendo intervenidos permanentemente, lo que se deriva en problemas de deforestación, pérdida de biodiversidad, uso irracional de aguas superficiales y subterráneas, alteración de la cadena trófica, destrucción de los sistemas naturales, alteración del paisaje y erosión del suelo.

Teniendo en cuenta lo anterior, en la empresa minera "El Porvenir" se beben implementar estrategias dirigidas a desarrollar actividades de las que se pueda tomar conciencia del daño que le causa este tipo de minería al medio ambiente producto de las prácticas inadecuadas que se desarrollan en estas. Esto, por consiguiente, puede mejorar significativamente los aspectos ambientales que están siendo afectados, también se podría implementar mecanismos de aprovechamiento sostenible de los residuos sólidos generados y la elaboración de un sistema convencional de tratamiento de las aguas residuales. 
Tabla 2: Matriz DOFA sobre diagnostico ambiental de la empresa "El Porvenir"

\begin{tabular}{|c|c|c|}
\hline \multirow{6}{*}{$\begin{array}{l}\text { Factores } \\
\text { Internos }\end{array}$} & Debilidades & Fortalezas \\
\hline & $\begin{array}{l}\text { Inadecuada disposición de residuos } \\
\text { sólidos. }\end{array}$ & $\begin{array}{l}\text { Condiciones climáticas, temperatura anual } \\
\text { elevada y pocas precipitaciones. }\end{array}$ \\
\hline & $\begin{array}{l}\text { No cuentan con sistema de tratamiento y } \\
\text { drenaje para las aguas provenientes del } \\
\text { lavado de la materia prima. }\end{array}$ & $\begin{array}{l}\text { Zonas con poca vegetación, favoreciendo } \\
\text { a la no tala de la flora. }\end{array}$ \\
\hline & $\begin{array}{l}\text { Inadecuada disposición de lodos que se } \\
\text { generan en el proceso del lavado del } \\
\text { yeso. }\end{array}$ & \\
\hline & $\begin{array}{l}\text { Captación ilegal de agua subterránea } \\
\text { para procesos de lavado. }\end{array}$ & \\
\hline & $\begin{array}{l}\text { No presentan iniciativas para la } \\
\text { búsqueda de soluciones a sus problemas } \\
\text { ambientales. }\end{array}$ & \\
\hline \multirow{4}{*}{$\begin{array}{l}\text { Factores } \\
\text { Externos }\end{array}$} & Oportunidades & Amenazas \\
\hline & $\begin{array}{l}\text { Entidades educativas interesadas por } \\
\text { conocer y buscar solución a las }\end{array}$ & $\begin{array}{l}\text { Contaminación de acuíferos, por causa del } \\
\text { vertido inadecuado de aguas residuales. }\end{array}$ \\
\hline & conalciones ambientales de la empresa. & $\begin{array}{l}\text { Proliferación de vectores a causa de la } \\
\text { inadecuada disposición de lodos y } \\
\text { residuos sólidos. }\end{array}$ \\
\hline & & $\begin{array}{l}\text { Contaminación atmosférica, por el cargue, } \\
\text { transporte y descargue de materiales } \\
\text { generados en la minería de yeso. }\end{array}$ \\
\hline
\end{tabular}

Tabla 3: Lista de chequeo de los impactos ambientales en la empresa "El Porvenir"

\begin{tabular}{|c|c|c|c|c|c|}
\hline \multirow{2}{*}{$\begin{array}{l}\text { Componentes } \\
\text { ambientales }\end{array}$} & \multirow{2}{*}{ Impactos } & \multicolumn{4}{|c|}{ Actividades de extracción y almacenamiento } \\
\hline & & $\begin{array}{l}\text { Extracción } \\
\text { de yeso }\end{array}$ & & & $\begin{array}{c}\text { Almacenamiento } \\
\text { de yeso }\end{array}$ \\
\hline \multirow{3}{*}{ Suelo } & Alteración del revestimiento vegetal & $X$ & & & $X$ \\
\hline & Perdida de suelo fértiles & & & & $X$ \\
\hline & Erosión & & & & $\mathrm{X}$ \\
\hline \multirow{3}{*}{ Flora y fauna } & Deforestación & & & & $\mathrm{X}$ \\
\hline & Pérdida de biodiversidad & & & & $X$ \\
\hline & Efectos en las especies endémicas & & & & $\mathrm{X}$ \\
\hline \multirow{3}{*}{ Territorio } & Uso inadecuado del territorio & & & & $X$ \\
\hline & $\begin{array}{l}\text { Modificaciones en el uso del } \\
\text { territorio }\end{array}$ & & & & $X$ \\
\hline & Expropiaciones del terreno & & & & $\mathrm{X}$ \\
\hline \multirow{2}{*}{$\begin{array}{l}\text { Alteración al } \\
\text { paisaje }\end{array}$} & Alteración del paisaje & $\mathrm{X}$ & & & $\mathrm{X}$ \\
\hline & $\begin{array}{l}\text { Destrucción de los sistemas } \\
\text { naturales }\end{array}$ & $X$ & & & $X$ \\
\hline \multirow[t]{2}{*}{ Aire } & $\begin{array}{l}\text { Emisión de gases de efecto } \\
\text { invernadero }\end{array}$ & & & & \\
\hline & Ruido ambiental & $\mathrm{X}$ & & & $\mathrm{X}$ \\
\hline \multirow{2}{*}{ Hombre } & Generación de empleo & $\mathrm{X}$ & & & $\mathrm{X}$ \\
\hline & Problemas a la salud & $\mathrm{X}$ & & & \\
\hline \multirow{2}{*}{$\begin{array}{l}\text { Componentes } \\
\text { ambientales }\end{array}$} & \multirow{2}{*}{ Impactos } & \multicolumn{4}{|c|}{ Actividades de lavado y molienda } \\
\hline & & Lavado de & & & rado del yeso \\
\hline \multirow[t]{3}{*}{ Suelo } & Inadecuados sistemas de drenaje & $X$ & & & \\
\hline & $\begin{array}{l}\text { Vertimientos permanentes de aguas } \\
\text { residuales }\end{array}$ & $X$ & & & $\mathrm{X}$ \\
\hline & Uso irracional de agua & $X$ & & & $\mathrm{X}$ \\
\hline
\end{tabular}


Tabla 3: continuación

\begin{tabular}{|l|l|c|c|}
\hline \multirow{2}{*}{$\begin{array}{l}\text { Componentes } \\
\text { ambientales }\end{array}$} & \multirow{2}{*}{ Impactos } & \multicolumn{2}{|c|}{ Actividades de lavado y molienda } \\
\cline { 2 - 4 } & & $\begin{array}{c}\text { Lavado de } \\
\text { yeso }\end{array}$ & Triturado del yeso \\
\hline \multirow{3}{*}{ Flora y fauna } & Poca disponibilidad de agua & $\mathrm{X}$ & \\
\cline { 2 - 4 } & Destrucción de hábitat & $\mathrm{X}$ & $\mathrm{X}$ \\
\cline { 2 - 4 } & Alteración de la cadena trófica & $\mathrm{X}$ & $\mathrm{X}$ \\
\hline \multirow{2}{*}{ Territorio } & Inestabilidad en el terreno & $\mathrm{X}$ & $\mathrm{X}$ \\
\cline { 2 - 4 } & Canales de drenaje & $\mathrm{X}$ & \\
\cline { 2 - 4 } & Extracción de la cobertura del terreno & $\mathrm{X}$ & $\mathrm{X}$ \\
\hline \multirow{2}{*}{$\begin{array}{l}\text { Alteración al } \\
\text { paisaje }\end{array}$} & Acumulación de lodos & $\mathrm{X}$ & $\mathrm{X}$ \\
\cline { 2 - 4 } Aire & Disposición inadecuada de residuos & & $\mathrm{X}$ \\
\hline \multirow{2}{*}{ Hombre } & Emisión de gases de efecto invernadero & $\mathrm{X}$ & $\mathrm{X}$ \\
\cline { 2 - 4 } & Ruido ambiental & $\mathrm{X}$ & $\mathrm{X}$ \\
\cline { 2 - 4 } & Generación de empleo & Problemas a la salud & \\
\hline
\end{tabular}

De acuerdo con los datos obtenidos a partir de la lista de chequeo, se puede determinar que la empresa minera "El Porvenir", existe diversos impactos ambientales producto de las actividades y/o procesos mineros que se llevan a cabo en esta mina, representando una alta probabilidad de riesgo ambiental y afectación en su área de influencia. Por tal motivo, se hace un llamado a la búsqueda de soluciones frente al daño ambiental que se genera en la empresa, puesto que actualmente el detrimento causado al medio ambiente es casi que imperceptible, pero se debe tener en cuenta que los efectos ambientales permanentes pueden ser irreversibles, provocando impactos graves en los ecosistemas; tal es el caso de los acuíferos, estos cuerpos de agua son fáciles de contaminar a través de las zonas de recarga, por infiltración o procesos kársticos producto de la disolución del yeso.

La entrada de yeso en el suelo y el agua puede representar amenazas potenciales para el medio ambiente y la seguridad alimentaria, al igual que generar el colapso de la mina. El estudio realizado por Sadeghiamirshahidi y Vitton (2019), muestra que el agua subterránea en minas de yeso abandonadas provoca la disolución de pilares de la roca hasta que el agua subterránea alcanza el potencial de saturación de yeso del agua subterránea, asimismo indican que en los sistemas de este tipo de minería a cielo abierto, cuando el agua subterránea sigue fluyendo a través de la mina, la disolución puede continuar; en esta situación, la resistencia de los pilares de las rocas se reduce, lo que da lugar a la inestabilidad de la mina y a un posible fallo (colapso), donde el tiempo hasta el fallo (si se produce) depende principalmente de la velocidad de disolución del yeso.

En efecto, se considera que una forma efectiva de contribuir positivamente en la empresa minera "El Porvenir", es mediante la implementación de estrategias ambientales que permitan la ejecución de buenas prácticas y utilización de herramientas alternativas; como, por ejemplo: la formulación y ejecución de prácticas favorables para el medio ambiente que podrían generarle ventajas al propietario de la empresa, por consiguiente, un impacto positivo para el medio ambiente.

\section{CONCLUSIONES}

A partir de los resultados obtenidos, se pueden extraer las siguientes conclusiones: 1) El estudio muestra que no existe una estrecha relación entre hacer uso eficiente de los recursos naturales y las actividades mineras; 2) "EI Porvenir" no desarrolla medidas de compensación ambiental, lo que quiere decir que la ejecución y puesta en marcha de los procesos de la empresa no contribuyen con el mejoramiento de los impactos ambientales negativos que esta genera; 3) es necesario diseñar medidas encaminadas a prevenir y mitigar los impactos ambientales que se han estado produciendo en la mina "El Porvenir"; 4) "El Porvenir" debe fomentar la creación y aplicación de programas de educación ambiental dirigidos a sus empleados y a las comunidades circundantes a la empresa; 5) la empresa debe diseñar una serie de planes de contingencia que les permita solventar alguna eventualidad imprevista. 6) La implementación de la matriz DOFA y la lista de chequeo son instrumentos eficaces para determinar los impactos generados a los componentes ambientales.

\section{REFERENCIAS}

Balnaves, M., y Caputi, P., Introduction to Quantitative Research Methods: An Investigative Approach, SAGE Publications Ltd., London, Great Britain (2001)

Cacilda-Andre, J., Lastra-Rivero, J., y Acevedo-Rodríguez, P., Impactos Ambientales de la Explotación Mecanizada de Materiales para la Construcción en Sumbe (Angola), Minería y Geología, ISSN: 1993-8012, 35(3), $338-357$ (2019) 
Canter, L.W., y Kamath, J., Questionnaire checklist for cumulative impacts, https://doi.org/10.1016/0195-9255(95)00010C, Environmental Impact Assessment Review, 15(4), 311-339 (1995)

Caselle, C., Bonetto, S.M.R., y Costanzo, D., Crack Coalescence and Strain Accommodation in Gypsum Rock, https://doi.org/10.3221/IGF-ESIS.52.19, Frattura Ed Integrità Strutturale, 14 (52), 247-255 (2020)

Cicatiello, L., Ercolano, S., Gaeta, G.L., y Pinto, M., Willingness to pay for Environmental Protection and the Importance of Pollutant Industries in the Regional Economy. Evidence from Italy, https://doi.org/10.1016/j.ecolecon.2020.106774, Ecological Economics, 177 (2020)

Creswell J.W., Research Design: Qualitative, Quantitative, and Mixed Methods Approaches, $2^{\mathrm{a}}$ edition, 1-246, SAGE Publiations Ltd., Londres, Nueva Delhi (2003)

Dong, Y.H., Ng, S.T., Kwan, H.K., y Wu, S.K., Substituting Local data for Overseas Life Cycle Inventories - a Case Study of Concrete Products in Hong Kong, https://doi.org/10.1016/j.jclepro.2014.10.005, Journal of Cleaner Production, 87, 414-422 (2015)

Fernandes, J.L., Qualharini, E.L., Nascimento, D.R., y Fernandes, A.S., Una Propuesta de Integración entre Licenciamiento Ambiental y Gestión de Proyectos en la Ciudad de Río de Janeiro-Brasil. http://dx.doi.org/10.4067/S0718-07642017000300002, Información tecnológica, 28(3), 3-16 (2017)

Figge, F. y Hahn, T., Business- and Environment-Related Drivers of Firms' Return on Natural Resources: A Configurational Approach, https://doi.org/10.1016/j.Irp.2020.102066, Long Range Planning, 1-19, (2020)

Fořt, J., y Černý, R., Carbon Footprint Analysis of Calcined Gypsum Production in the Czech Republic, https://doi.org/10.1016/j.jclepro.2018.01.002, Journal of Cleaner Production, 177, 795-802 (2018)

Gunnarsdottir, I., Davidsdottir, B., Worrell, E. y Sigurgeirsdottir, S., Sustainable Energy Development: History of the Concept and Emerging Themes, https://doi.org/10.1016/j.rser.2021.110770, Renewable and Sustainable Energy Reviews, 141 (2021)

Guo, X.L. y Shi, H.S., Thermal Treatment and utilization of Flue Gas Desulphurization Gypsum as an Admixture in Cement and Concrete, https://doi.org/10.1016/j.conbuildmat.2007.04.001, Construction and Building Materials, 22(7), 1471-1476 (2008)

He, X., Yuan, T., y otros 3 autores, Effects of Contaminated Surface Water and Groundwater from a rare Earth Mining Area on the Biology and the Physiology of Sprague-Dawley rats, https://doi.org/10.1016/j.scitotenv.2020.144123, Science of The Total Environment, 761 (2021)

Hernández, R., Fernández, C. y Baptista, P., Metodología de la Investigación, Mc Graw Hill, México (2010)

Hilson, G., y Nayee, V., Environmental Management System Implementation in the Mining Industry: a key to Achieving Cleaner Production, https://doi.org/10.1016/S0301-7516(01)00071-0, International Journal of Mineral Processing, 64(1), 19-41 (2002)

Ikram, M., Zhou, P., Shah, S.A., y Liu, G.Q., Do Environmental Management Systems help Improve Corporate Sustainable Development? Evidence from Manufacturing Companies in Pakistan, https://doi.org/10.1016/j.jclepro.2019.03.265, Journal of Cleaner Production, 226, 628-641 (2019)

Jiménez-Rivero, A., y García-Navarro, J., Indicators to Measure the Management Performance of End-of-Life Gypsum: From Deconstruction to Production of Recycled Gypsum, https://doi.org/10.1007/s12649-016-9561-x, Waste Biomass Valor, 7, 913-927 (2016)

Li, H., Lo, K., y Wang, M., Economic Transformation of Mining Cities in Transition Economies: Lessons from Daqing, Northeast China, https://doi.org/10.3828/idpr.2015.19, Int. Development Planning Review, 37(3), 311-328 (2015)

Lo, K., Ecological Civilization, Authoritarian Environmentalism, and the Eco-politics of Extractive Governance in China, https://doi.org/10.1016/j.exis.2020.06.017, The Extractive Industries and Society, 7(3),1029-1035 (2020)

López, D., Mucho más que Carbón, el Escenario Minero de La Guajira, 1-140, Gente Nueva, Riohacha, Colombia (2010)

Molléri, J.S., Petersen, K., y Mendes, E., An Empirically Evaluated Checklist for Surveys in Software Engineering, https://doi.org/10.1016/j.infsof.2019.106240, Information and Software Technology, 119 (2020)

Nikolaou, I.E., y Evangelinos, K. I., A SWOT Analysis of Environmental Management Practices in Greek Mining and Mineral Industry, https://doi.org/10.1016/j.resourpol.2010.02.002, Resources Policy, 35(3), 226-234 (2010)

Ricci, J.L., Alexander, H. y otros 7 autores, Biological Mechanisms of Calcium Sulfate Replacement by bone, Bone Engineering, 332-344, Em Squared Inc., Toronto, Canada (2000)

Ross, M.L., What do we know about Export Diversification in Oil-Producing Countries?, https://doi.org/10.1016/j.exis.2019.06.004, The Extractive Industries and Society, 6(3), 792-806 (2019)

Sadeghiamirshahidi, M., y Vitton, S.J., Laboratory Study of Gypsum Dissolution Rates for an Abandoned Underground Mine, https://doi.org/10.1007/s00603-018-1696-6, Rock Mechanics and Rock Engineering, 52, 2053-2066 (2019)

Stavru, S., A Critical Examination of Recent Industrial Surveys on Agile Method Usage, https://doi.org/10.1016/j.jss.2014.03.041, Journal of Systems and Software, 94, 87-97 (2014) 